\title{
The expanding toolbox for genome engineering
}

\author{
The optimization and diversification of methods for manipulating the genome will enable new therapeutic solutions.
}

$\mathrm{T}$ he fast pace of preclinical research in genome engineering is bringing its potential applications in human health closer to reality. Gene-editing technologies, and in particular methods using CRISPR (clustered regularly interspaced short palindromic repeats), which are based on RNA-guided bacterial nucleases that cut DNA at specific locations and induce the removal or replacement of genes, have been employed to modify patient-derived cells ex vivo, especially for cell-based cancer immunotherapy. Recently, CRISPR-based DNA editing has corrected diseaseassociated mutations in human embryos (H. Ma, et al. Nature 548, 413-419; 2017). Such steps towards the clinical translation of genome-engineering technologies raise a whole new set of challenges, from ethical considerations to limitations in the efficiency, safety and specificity of the genome-modification approaches.

The first attempts at CRISPR-mediated DNA editing with the Cas9 (CRISPRassociated protein 9) endonuclease had low efficiency (that is, only a small subset of cells were modified) and insufficient specificity (DNA sites other than the intended targets were altered). Low efficiency is not always a problem, as therapeutic effects can sometimes be achieved when only a fraction of the target cells are successfully edited. Issues with specificity, however, need to be thoroughly addressed if genome-editing technologies are to be used in human therapies. To this end, Cas9 and other endonucleases, as well as the guide RNA (gRNA), can be modified to enhance the specificity and efficiency of editing.

Cells repair DNA breaks mainly through the endogenous non-homologous end joining (NHEJ) DNA-repair pathway, a process that can introduce or delete nucleotides at the DNA-break region and that is therefore amenable to permanent silencing of target genes. Before entering mitosis, cells can also repair double-strand breaks by homology-directed repair (HDR), a more accurate mechanism involving homologous recombination in the presence of a template DNA strand. In this issue, Derrick Rossi and colleagues report conditions that favour HDR rather than NHEJ. By screening DNA-repair factors, the researchers found that inducing the ectopic expression of RAD52 (a DNA-repair factor involved in homologous recombination) and of a dominant-negative form of 53BP1 (another DNA-repair factor) led to increased HDR efficiency at targeted loci in induced pluripotent stem cells. Importantly, the enhanced HDR efficiency did not come at the expense of specificity, as the rate of offtarget activity remained unaltered. Gaining increased control over the accuracy of gene editing will eventually speed up the translation of these techniques for the correction of disease-causing mutations in patients.

Before genes can be corrected through HDR-based gene editing in vivo, all components of the CRISPR machinery typically Cas9, gRNA and donor template DNA - need to be delivered to the right cells. Viral vectors, such as adeno-associated viruses (AAVs), can deliver genes and CRISPR components to target cells in preclinical models of disease. However, AAVs are not amenable to broad clinical use because a large proportion of the population has immunity against these viruses. Also, the small size of AAVs limits the number of components that can be encapsulated, as well as the extent of control over the expression and activity of Cas9 when the gene rather than the protein is delivered. Alternatively, nanoparticles can deliver a wide variety of molecules to target cells in vivo. This is the approach used by Niren Murthy and colleagues, who report that Cas9 protein, gRNA and template DNA can be attached to gold nanoparticles (these were then complexed with endosomal disruptive polymers to facilitate release into the cytoplasm after the nanoparticles are internalized by cells) to effectively deliver the molecules in vitro and in vivo, facilitating the editing of genes via HDR. The injection of the loaded nanoparticles in a mouse model of muscular dystrophy led to the correction of a disease-causing mutation in the dystrophin gene expressed in muscle tissue, and to improved muscle function in the treated animals. Also, off-target DNA damage was minimal, and multiple injections did not elevate the levels of inflammatory cytokines in blood plasma and did not cause the animals to lose weight.

In addition to the use of CRISPR methods, the genome can also be manipulated at the level of chromatin, and

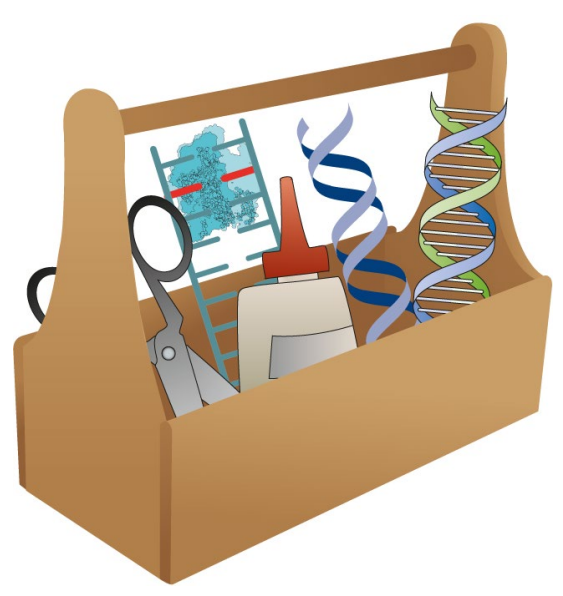

targeted chromatin modifications that affect gene-expression patterns may also lead to approaches for tackling disease. This rationale has led to the use of a range of histone deacetylase inhibitors to modify the epigenome at the chromatin level, for cancer treatment. Vadim Backman and colleagues demonstrate that the response of cancer cells to chemotherapy can be enhanced with molecules that reduce the intracellular heterogeneity of the packing density of chromatin (thereby limiting the amount of available genomic information that cancer cells can explore, which contributes to chemoevasion), and propose that the direct control of the chromatin nanoenvironment can be exploited to manipulate the cell's transcriptome, as well as intercellular transcriptional heterogeneity, to combat disease. Such reversible macrogenomic engineering could complement gene-editing approaches.

The ever-improving knowledge of the organization and composition of the genome and the epigenome, together with the expanding technological toolbox for the manipulation of DNA with increasing precision, are propelling the exploration of how genome-engineering approaches can be translated to tackle diseases that escape conventional therapies. That's a boon for translational research and, ultimately, the patients.

Published online: 10 November 2017 https://doi.org/10.1038/s41551-017-0163-0 